\title{
50 Jahre danach - sind wir schon weiter?
}

\section{Zur Ausgangslage}

Wer als Theolog^in zu ökumenischen Vorträgen und Gesprächsabenden an der Basis unterwegs ist, dem/der wird häufig entgegengehalten: „Wir sind aber schon viel weiter als die [...]“. Nun gilt hier in besonderem Maße, was jedem theologischen Statement gut ansteht: differenzieren! Gewiss, man kann sich auch totdifferenzieren und dann leicht sarkastisch feststellen: „Gut, dass wir darüber geredet haben." Es ist nicht ganz von der Hand zu weisen, dass auch für die ökumenische Szenerie gilt: „Wir haben jetzt lange genug interpretiert, nämlich Lehrverurteilungen aufgearbeitet und Bekenntnistexte miteinander verglichen - nun geht es darum auch zu handeln!“ Gleichwohl müssen Theolog*innen einen gewissen Widerstand leisten und auf das Unterscheiden, griech: das krinein (der Wortstamm für Krise und Kriteriologie), drängen. Bildet das folgenlose und immer wieder aufs Neue eingeforderte „das müssen wir nochmal gründlich aufarbeiten“ den einen, so der heillose Aktivismus den anderen Pol des ökumenischen Spektrums.

Was gilt es zu unterscheiden? Ganz grob, notwendig wenn auch nicht hinreichend, sind es die drei Großsubjekte kirchlich-theologischer Kommunikation: das Gottesvolk insgesamt, zu dem wissenschaftlich arbeitende Theolog*innen sowie Repräsentant*innen in mehr oder weniger lehramtlicher Funktion dazugehören - und das nicht in einem Darüberstehen, sondern einem verantwortlichen Miteinander. Die Zuordnung dieser drei Bezeugungsinstanzen (Gemeinden/ Kirchen-,Lehramt“-wissenschaftlich arbeitende Theologie) zueinander und dann vor allem zu den normativen Bezeugungsinstanzen, vor allem zur Auslegung der Heiligen Schrift, ist selbst wieder in den Konfessionskirchen unterschiedlich ausgeprägt. Ich erinnere nur an das geradezu konträre Procedere im Zusammenhang der Gemeinsamen Erklärung zur Rechtfertigungslehre. Inwieweit auf evangelischer Seite Mitchristinnen, die keine Theolog`innen sind und kein Kirchenamt begleiten, jeweils involviert sind, wird selbst differenziert zu prüfen sein. Katholischerseits jedenfalls klafft eine Kommunikationslücke zwischen Lehre und Theologie auf der einen und den Erfahrungen und der Praxis der Gläubigen in den Gemeinden. Ja, wie Theodor Schneider, einer der Altmeister ökumenischer Theologie, feststellt, gilt diese Kluft auch für das Verhältnis von Lehramt und Theologie - und das eben nicht nur in synchroner, sondern auch in diachroner 
Hinsicht. ${ }^{1}$ Wer, auch von den heutigen Bischöfen, kennt die ökumenischen Dokumente, die in Jahrzehnten erarbeitet wurden? Wer kennt das Ökumenismusdekret und überhaupt die Texte des Zweiten Vatikanischen Konzils, von der Würzburger Synode ganz zu schweigen? Und wie steht es mit der Kenntnis der in Lima verabschiedeten Konvergenzerklärungen, des Projekts Lehrverurteilungen kirchentrennend?, wie mit der Gemeinsamen Erklärung, wie mit Vom Konflikt zur Gemeinschaft? Gewiss gilt auch hier das notwendige Unterscheiden: innerhalb der drei Großsubjekte gibt es selbst ein breites Spektrum an bewusst vertretenen oder auch nur de facto eingenommenen Positionen. Hier gilt, was Johanna Rahner unlängst öffentlich und deutlich behauptete: Die Furcht vor Kirchenspaltung ist unbegründet, denn wir haben sie schon. Das erschwert das ohnehin nicht einfache Amt episkopal-lehramtlicher Prägung und erzwingt die Verabschiedung eines undifferenzierten Einheitsdenkens, das möglichst una voce zum Ausdruck gebracht werden soll.

Die Frage also, ob wir 40 Jahre nach der einschlägigen Debatte und zur Entlastung der kommenden 10 Jahre behaupten dürfen, wir seien schon (viel) weiter, kann nur differenzierend beantwortet werden, wobei ich jetzt nur für die römisch-katholische Seite spreche. Ökumenisch engagierte und z. B. bewusst eine konfessionsverbindende Ehe lebende Katholik`innen schätzen ihre evangelischen Partner*innen, bewundern Charakteristika ihres Glaubenslebens, gönnen ihnen die Freiheit im Kompetenzennetz der Glaubenskommunikation. Diese Katholik^innen wollen zugleich ihre eigene Herkunft einbringen und lebendig halten oder auch wieder erwecken, und sie fragen sich, ob die unterschiedlichen Lehrgebäude, von denen sie zumeist nur gehört, die sie aber nie gelehrt und erklärt bekamen, ihr gemeinsames Christenleben spalten kann. Zwei Reaktionen von Seiten des Lehramtes und der Theologie sind in jedem Fall denkbar: entweder das Projekt einer großangelegten, aber wohl kaum noch flächendeckend gefragten ökumenischen Katechese und Erwachsenenbildung oder der Versuch, die Erfahrungen der Basischristen ernst zu nehmen und auf ihre Relevanz für die Einschätzung der womöglich gar nicht mehr kirchentrennenden unterschiedlichen Lehrgestalten zu befragen.

Die römisch-katholische Kirche hat ihre eigene Geschichte, was das Verhältnis von Glaubenslehre und Glaubenserfahrung angeht. Selbst da, wo das sich auf die eigene Erfahrung Berufen nicht absolut gesetzt wurde und nicht seinerseits Kommunikation verweigerte, begegnete Argwohn und Abwertung. Spätestens an dieser Stelle wird deutlich, dass es einschlägige Machtverhältnisse in der kon-

1 Vgl. Theodor Schneider, Ökumenisches Porträt, in: Una Sancta 71 (2016), 71-79. 
kreten Ausprägung des Kommunikationsdreiecks ${ }^{2}$ gibt, und es gilt ins Auge zu fassen, dass der ekklesiologischen und von daher auch der ökumenischen „Psychologie“ größeres Gewicht zukommt als Ekklesiologie und ökumenischer Theologie. ${ }^{3}$

Beim Niederschreiben habe ich mich, spätestens an dieser Stelle, gefragt, ob die Bedenken, die ich für notwendig erachte, überhaupt hilfreich sein können für die Tagung selbst und das Projekt der ökumenischen Bemühungen um die Confessio Augustana. War die Situation nicht immer so, so dass es keinen Sinn macht, hier als ökumenische Greta Thunberg aufzutreten und endlich schnelles Handeln einzufordern, bevor das ökumenische Klima kollabiert? Es mag sein, dass sich etwa durch den Beitrag von Theo Dieter herausstellt: wir sind heute nicht weiter als in den 70er Jahren und um 1980 herum. Wenn wir aber, was den theologischen Stand angeht, nicht weiter wären (was ich nicht für zutreffend halte), so hat sich gleichwohl der Kontext, der kirchliche und gesamtgesellschaftliche, erheblich verändert. Und heutzutage leugnet ja kein vernünftiger Theologe, dass Kontextualität von elementarer Bedeutung für die Verantwortung des überlieferten Glaubens ist. Damit betreibt ökumenische Theologie keine der einseitigen Formen von Ökumene, die seinerzeit Kardinal Ratzinger den Thesen von Heinrich Fries und Karl Rahner ${ }^{4}$ bzw. ihrer Stoßrichtung unterstellte: ${ }^{5}$ Es handelt sich weder um eine „Basisökumene“, die die Gemeinden spaltet, noch um eine die Bischöfe bloß belehrende und von oben herab dozierende „Obrigkeitsökumene“, noch ist Ökumene Verhandlungssache - ein Vorwurf, den Joseph Ratzinger als Benedikt XVI. erneut erhoben hatte. Mir scheint, dass es sich lohnt, folgende Spur zu verfolgen, die ich in diesem Rahmen nur vorsichtig vorzeichnen kann: Wenn Christenmenschen ihren Glauben im ökumenischen Miteinander leben und sich im Kern nicht als getrennt erfahren, wenn der geänderte gesellschaftliche Kontext - zunehmende Kirchenaustritte bzw. Nicht-eintritte, so dass die Christ^innen nicht mehr die Mehrheit in der Bevölkerung darstellen; Herausforderung durch andere Religionen und Religionsformen, besonders durch die Muslim^innen in unserem Land; gemeinsame Herausforderungen ethischer Art und das Wach-

2 Vgl. meinen Beitrag: Das Spannungsverhältnis zwischen Kirchenleitung, Theologie, Basis und geistlichen Gemeinschaften aus römisch-katholischer Perspektive, in: Una Sancta H.3/2013 (Themenheft), 195-205.

3 Vgl. meinen Beitrag: Nicht-genuin-theologische Faktoren im Prozess der (Ent)Konfessionalisierung. In Erinnerung an Günther Gassmann, in: Theologische Quartalschrift 197 (2017), 277288.

4 Heinrich Fries/Karl Rahner, Einigung der Kirchen - reale Möglichkeit, Freiburg i.B. 1983.

5 Vgl. Heinrich Fries, Einigung der Kirchen - reale Möglichkeit, in: Heinrich Fries/Otto Hermann Pesch (Hg.), Streiten für die eine Kirche, München 1987, 77-81. 
halten einer Zukunftshoffnung - wenn dieser Kontext dringender denn je, wenn auch spezifischer zur Besinnung auf das, was wir gemeinsam bezeugen können, zwingt, was bedeutet dies dann für das Verhältnis der Konfessionskirchen zueinander? Müssen wir wieder einmal gezielt das Verhältnis von sprechendem und handelndem Glauben, von Orthodoxie und Orthopraxie ins Auge fassen? ${ }^{6}$ Wieweit kann uns eine mögliche Anerkennung der CA bei diesem Projekt helfen?

\section{Zur Struktur der Glaubenslehre}

Melanchthons Anliegen war es, durch die Unterscheidung von unverzichtbaren Fundamentalartikeln - systemisch gesprochen: von solchen, die Unterschiede machen würden - und den Adiaphora, also Artikeln, denen kein kirchentrennender Charakter zukommt, den Beweis zu erbringen, dass die Reformwilligen und die Altgläubigen im Herzen des Glaubens übereinstimmen. Manche Artikel würden auch heute noch so eingeordnet sein, andere hinzukommen oder an die Stelle treten. Darüber ging ja vor 40-50 Jahren die Debatte, wie Theo Dieter zeigen wird, und genau diese zuordnende Unterscheidung spielte auch in der Rezeption der Debatte in nachfolgenden ökumenischen Dokumenten eine Rolle. Nach Jahrzehnten macht es keinen Sinn einfach zu kopieren; zumindest in diesem Sinn würde eine Anerkennung der CA gerade keinen Sinn ergeben. Geradezu mustergültig ist dagegen die Unterscheidung zwischen gemeinsamem Fundament und legitimer Pluralität im Sinn eines differenzierenden Konsenses. Einige Beiträge hier haben sich ja schon mit der Frage auseinandergesetzt, welche Relevanz den damaligen Kontroversen im Einzelnen heute noch zukommt. Zugleich ist zu fragen, welche damals so nicht artikulierten Lehrunterschiede heute einer Anerkennung der Kirchen als legitime Ausprägungen der einen Kirche Jesu Christi im Wege stünden bzw. übereinstimmend als nicht mehr kirchentrennenden eingestuft werden können. Wie uns hier in Erinnerung gerufen wurde, sind die Topoi der CA: Gott und der Mensch vor Gott und der Weg des Menschen in und mit der Kirche, mit den Heilsmitteln zu Gott. In einer tatsächlichen oder auch nur, jedenfalls partiell, nominell christlichen Welt kommt der Vielzahl der Heilsmittel auf dem einen Heilsweg mit Jesus Christus viel Aufmerksamkeit zu. Heute scheint eine Konzentration auf den, den wir als "den Weg, die Wahrheit und das Leben“ bekennen, Vorrang zu haben. Es geht im Kontext der interreligiösen Begegnung wie eines neuen Atheismus, Agnostizismus und Desperantismus darum, die Au-

6 S. jetzt: Theodor Schneider, Orthodoxie und Orthopraxie. Glauben bekennen - Glauben leben, hg. u. weitergeführt von Bernd Jochen Hilberath/Dorothea Sattler, Ostfildern 2020. 
gen $z u$ öffnen für und die Hoffnung wachzuhalten auf den Gott, der - ohne aufzuhören Gott zu sein - in diese Menschenwelt eingeht, auf die Menschen zugeht und mit ihnen geht. In Anlehnung an Karl Rahner lässt sich formulieren: Unser transzendierendes Sehnen nach seliger Erfüllung verfehlt nicht asymptotisch sein Ziel. Wir geben Zeugnis vom „Grund unserer Hoffnung“ - ich zitiere bewusst das Bekenntnis der Würzburger Synode, die ja im Jahrzehnt vor dem CAJubiläum ebenfalls den Versuch wagte, die Fundamentalartikel unseres Glaubens im Heute zu formulieren. ${ }^{7}$ Wir Christinnen - und das interreligiöse Gespräch wird erweisen, wieweit dies auch für andere Religionen gilt - glauben, einen Grund benennen zu können, weshalb unser Streben nicht ins Leere greift: Gott selbst ist transzendent und unverfügbar, aber aus freien Stücken - wir nennen es Gnade, also sola gratia - nicht in asymptotischer Fastannäherung geblieben. „Wer mich gesehen hat, hat den Vater gesehen“ (Joh 14,9) ist das Herzstück des Evangeliums. Wir Christ*innen können von Gott nur sprechen, wenn wir in dieser Theo-logik von Jesus Christus sprechen.

So wenig wie zu Zeiten Melanchthons - von Ausnahmen abgesehen - Gott in Frage gestellt wurde, so wenig war auch das trinitarische Gottesbild bestritten, jedenfalls weithin. Heute erfordert die Kontextualität unseres Glaubens ein entsprechendes Zeugnis. Es genügt eben nicht, sich mit dem mehr oder weniger gewissen Festhalten an dem allmächtigen und barmherzigen Gott zufrieden zu geben, jedenfalls genügt dies für das unverkürzte und evangeliumsgemäße Lehren der Kirchen nicht. Aber nicht primär um der Integrität willen, sondern in erster Linie um des menschenfreundlichen Gottes willen bedeutet Rechenschaft vom Grund unserer Hoffnung heute, von Gott, Jesus Christus und, was in der CA keine Rolle spielt, dem Heiligen Geist zu sprechen in Leiturgia, Martyria und Diakonia.

Die römisch-katholische Kirche war in dieser Hinsicht zwar nicht weiter, aber immerhin bis dahin gelangt. In der Debatte um das Ökumenismusdekret spielte die Rede von der Hierarchie der Wahrheiten eine gewichtige Rolle (und die Würzburger Synode hat sie in ihrem Beschluss zur Ökumene 3.2.4 aufgenommen). ${ }^{8}$ Auf dem Konzil ${ }^{9}$ wurde diese methodologische Orientierung eingebracht von Bischof Pangrazio aus Gorizia, der in einem multikulturellen, inhomogenen

7 Unsere Hoffnung. Ein Bekenntnis zum Glauben in dieser Zeit, in: Gemeinsame Synode der Bistümer in der Bundesrepublik Deutschland. Offizielle Gesamtausgabe (I), Freiburg i.B. 1976, 84-111 (mit einer Einleitung von Theodor Schneider, 71-84).

8 Pastorale Zusammenarbeit der Kirchen im Dienst an der christlichen Einheit, in: Gemeinsame Synode, 774-806, hier: 781 (Einleitung von G. Voss, 765-773).

9 S. dazu meinen Kommentar zum Ökumenismusdekret „Unitatis Redintegratio“, in: Herders Theologischer Kommentar zum Zweiten Vatikanischen Konzil, Bd. 3, Freiburg i.B. 2005, 69-223. 
Kontext lebt, was möglicherweise „seine ungewöhnliche ökumenische Nachdenklichkeit“10 erklärt. Später sorgte dann Kardinal König von Wien für die erneute Aufnahme dieses Theologumenons. Pangrazio beklagte, dass der Aufzählung der elementa ecclesiastica das „einigende Band“ und Zentrum fehle, nämlich Jesus Christus, „den alle Christen als Herrn der Kirche bekennen, dem zweifellos die Christen aller Gemeinschaften treu dienen wollen und der auch in den getrennten Gemeinschaften durch seine aktive Gegenwart im Heiligen Geist seine Wunder vollbringen will, freilich nicht durch die Verdienste der Menschen, sondern allein durch die Gnade seiner Barmherzigkeit“"11.

Ich möchte vor dem Hintergrund der nachkonziliaren Nicht-Wirkungsgeschichte weiter fragen: Handelt es sich nicht dann bei den „Gemeinschaften“ um „Kirchen im eigentlichen Sinn“? Nach Pangrazio ergibt sich aus der Struktur der Offenbarung selbst eine Hierarchie der Wahrheiten, derzufolge die Wahrheiten des Zieles über den Wahrheiten der Mittel einzuordnen sind. $\mathrm{Zu}$ den ersten gehört „das Geheimnis der allerheiligsten Dreifaltigkeit, der Menschwerdung des Wortes und der Erlösung, der göttlichen Liebe und Gnade gegenüber der sündigen Menschheit, des ewigen Lebens in der Vollendung des Reiches Gottes“12. Zu den sekundären Wahrheiten der Heilsmittel zählt Pangrazio z.B. „die Wahrheiten über die Siebenzahl der Sakramente, über diese hierarchische Ordnung der Kirche, über die apostolische Sukzession“. Dies seien Mittel für den „irdischen Pilgerweg“ der Kirche, aber nicht ewige Wahrheiten.

1990 veröffentlichte eine Gemeinsame Arbeitsgruppe des Päpstlichen Rates zur Förderung der Einheit der Christen und der Kommission für Glauben und Kirchenverfassung ein Studiendokument unter dem Titel „Der Begriff der ,Hierarchie der Wahrheiten " - eine ökumenische Interpretation“13. Eine hoffnungsvolle Perspektive tut sich auf, wenn es darin heißt: „Eine Anerkennung der ,Hierarchie der Wahrheiten' könnte bedeuten, dass die ökumenische Tagesordnung auf eine Gemeinschaft im ,Fundament' gegründet wird, die bereits besteht und den Weg weisen wird $\mathrm{zu}$ jener Ordnung von Prioritäten, die ein allmähliches Hineinwachsen in die volle Gemeinschaft ermöglicht. “14

Die Gemeinsame Erklärung war als wichtiger Schritt auf diesem Weg gedacht, als Meilenstein, der dann für manche zum Stolperstein wurde oder dafür erklärt wurde. Ich frage mich an dieser Stelle und gebe die Frage weiter: Sind wir nicht

10 Otto Hermann Pesch, „Hierarchie der Wahrheiten“ - und die ökumenische Praxis, in: Concilium 37 (2001), 310, Anm. 13.

11 AS (Acta Synodalia) II/6, 34.

12 AS (Acta Synodalia) II/6, 34.

13 In: DwÜ (Dokumente wachsender Übereinstimmung) 2, 751-760.

14 DwÜ 2, 758. 
längst in die volle Gemeinschaft hineingewachsen, so dass wir das „allmählich“ streichen können, auch wenn eine ,Gemeinsame Offizielle Feststellung‘ noch aussteht?

Unter uns sind Berufenere, die aufzeigen können, wie diese Unterscheidung einer Hierarchie der Wahrheiten in den ökumenischen Dokumenten seit 1980 sichtbar wird. Sind wir nicht inzwischen „Vom Konflikt zur Gemeinschaft“ gekommen? Hat nicht z. B. das Plädoyer der drei Ökumenischen Institute Abendmahlsgemeinschaft ist möglich ${ }^{15}$ vor fast 20 Jahren vorweggenommen, was jetzt vom ÖAK ausführlich dargelegt und als „theologisch ausreichend“ beurteilt wird? Wir können nur hoffen, dass die Kritik jetzt differenzierter ausfällt, zumal es ja nicht gleich zu sein scheint, wer was in Sachen Ökumene vorträgt - das wäre dann wieder ein Thema der Ökumenischen Psychologie. Ich will an dieser Stelle die Thesen von Fries und Rahner unter dem Gesichtspunkt der Struktur der Glaubenslehre in Erinnerung rufen. ${ }^{16}$

Die erste These lautet: „Die Grundwahrheiten des Christentums, wie sie in der Heiligen Schrift, im Apostolischen Glaubensbekenntnis und in dem von Nizäa und Konstantinopel ausgesagt werden, sind für alle Teilkirchen der künftig einen Kirche verpflichtend. “17

Schnell hören wir die Einwände: Was sind denn die biblischen Grundwahrheiten und vor allem: wer legt sie verbindlich aus und vor? Genügt das Zitieren des Symbolums, selbst wenn das in der Liturgie geschieht? Teilen denn alle das gleiche Verständnis der Grundwahrheiten? Wir erinnern uns an die Debatte um die Gemeinsame Erklärung zur Rechtfertigungslehre: „Konsens in Grundwahrheiten“, also nicht dezidiert „in den Grundwahrheiten“. Und jetzt einen Konsens in Grundwahrheiten nicht nur der Rechtfertigungslehre, sondern aller fundamentalen Glaubensartikel? Diese kritischen Nachfragen sind nicht überflüssig und nicht beckmesserisch, jedoch: Wie kann es jemals zur Feststellung eines differenzierenden Konsenses kommen, wenn immer und bei allem alle mitgenommen werden müssen und quasi die Katechismusprüfung $\mathrm{zu}$ bestehen haben? Die Hierarchie der Wahrheiten des je persönlichen Glaubens muss nicht de-

15 Centre d'Études Ecuméniques (Strasbourg)/Institut für Ökumenische Forschung (Tübingen)/ Konfessionskundliches Institut (Bensheim), Abendmahlsgemeinschaft ist möglich. Thesen zur Eucharistischen Gastfreundschaft, Frankfurt a. M. 2003, ${ }^{2} 2007$.

16 Fries/Rahner, Einigung.

17 Fries/Rahner, Einigung, 17. 
ckungsgleich sein mit dem, was die Glaubensgemeinschaft als solche festhält, es sei denn, das laufe auf die Leugnung von Grundwahrheiten hinaus. ${ }^{18}$

Ich möchte diese kritischen Nachfragen, die ja auch unser Geschäft als ökumenische Theolog^innen betreffen, zurückstellen. Das wäre nur dann fatal, wenn eine Einheit der Kirchen in versöhnter Verschiedenheit allein auf der Basis eines vollständigen (fach)theologischen Konsenses erklärt werden könnte. Freilich liebäugele ich nicht mit einer Ökumene der Differenz, die ja nur spiegelbildlich als vollständig gegeben abbilden müsste, dass es eben keinen Konsens in Grundwahrheiten geben könne. Der freilich nur grob skizzierte Kontext unseres heutigen Glaubens, Lehrens und Theologietreibens empfiehlt das, was Dorothea Sattler ihrem Statement zur jüngsten ÖAK-Studie als Überschrift gewählt hat, eine Hermeneutik des Vertrauens. ${ }^{19}$ Dies muss, bis zum öffentlichen Erweis des Gegenteils, einschließen, dass alle, die zu einer Kirche gehören, die grundlegenden Überzeugungen dieser Gemeinschaft zumindest implizit annehmen und dass die, die für die theologische Reflexion zuständig sind bzw. den Lehrkonsens der je eigenen Kirche vertreten, es als ausreichend erachten, dass sich die jeweiligen Theologien und Lehrgebäude der Bekenntnisschriften und Dogmen prinzipiell auf die Schrift und die altkirchlichen Bekenntnisse beziehen wollen. Übrigens sind diese Erwägungen und Bedenken schon in den Kommentaren von Fries und Rahner berücksichtigt worden, und sie haben die Formulierung der zweiten These mitbestimmt: „Darüber hinaus gelte ein realistisches Glaubensprinzip: in keiner Teilkirche darf dezidiert und bekenntnismäßig ein Satz verworfen werde, der in einer anderen Teilkirche ein verpflichtendes Dogma ist." Und abschließend wird hinzugefügt: „Bei diesem Prinzip würde nur getan, was jede Kirche heute schon ihren eigenen Angehörigen gegenüber praktiziert." ${ }^{20}$ Die Thesen von Fries und Rahner wurden z.T. heftig von zwei entgegengesetzten Seiten kritisiert, prominent von Eilert Herms und offiziös im Osservatore Romano (von Daniel Ols OP) ${ }^{21}$ Eine gewisse Allianz verbindet offenbar einen Typ dezidierten Luthertums mit einem Typ konservativen Katholizismus; da sind beide Positionen klar und man weiß, wogegen man seine eigene Identität verteidigen muss. Dass dann im Zusam-

18 Hellsichtig und zukunftsweisend auch in dieser Frage: Karl Rahner, Offizielle Glaubenslehre der Kirche und faktische Gläubigkeit des Volkes, in: Schriften zur Theologie, Bd. 16, Zürich/ Einsiedeln/Köln 1984, 217-230.

19 KNA-ÖI 38 v. 17.09.2019.

20 Fries/Rahner, Einigung, 17.

21 Vgl. dazu den Kommentar von H. Fries, in: Fries/Pesch, Streiten für die eine Kirche, 51-61 (zu Herms) und 61-68 (zu Ols). 
menhang des mit der Lateranuniversität durchgeführten Studienprojekts ${ }^{22}$ behauptet wurde, hier werde endlich die richtige Methodologie ökumenischer Theologie (vielleicht doch zutreffender: Kontroverstheologie) angewendet, verblüfft bzw. spricht eben Bände!

Mit Blick auf die einschlägige Diskussion schreibt Heinrich Fries:

Unsere These I sagt dagegen, in den grundlegenden Inhalten des christlichen Glaubens, bezeugt in der Heiligen Schrift, bestätigt in den alten Glaubensbekenntnissen und Konzilien, gäbe es eine große verbindende Gemeinsamkeit. Davon geht auch die Confessio Augustana von 1530 aus, ebenso Luther in den Schmalkaldischen Artikeln, wo er erklärt, in den ,hohen Artikeln der göttlichen Majestät, im Glauben an den dreifaltigen Gott und an Jesus Christus gebe es keinen Zank und Streit, weil wir zu beiden Seiten dasselbe glauben und bekennen'. Auf der Gemeinsamkeit in den Grundartikeln beruht die Basisformel des Weltrats der Kirchen, ebenso [berufen darauf] sämtliche ökumenische Dokumente [... $]^{23}$.

Ich kann nachvollziehen, dass evangelische Kolleg*innen insbesondere beim Lesen der zweiten These geschluckt haben. Das dogmatische Gebäude der römisch-katholischen Kirche erscheint nun als unvergleichbar größer oder höher, während auf evangelischer Seite sogar die Zustimmung zu den Bekenntnisschriften nicht überall gleich verpflichtend gemacht wird oder de facto bindend ist. Akzeptabler erscheint mir eine Fassung dieser These, die lautet: „Keine Kirche sollte eine Lehre als für alle verbindliche vertreten, die nach dem Urteil anderer Kirchen dem Konsens in Grundwahrheiten widerspricht und die Identität des Christlichen gefährdet." Unter Berücksichtigung der Hierarchie der Wahrheiten könnte der Satz auch heißen: „die nach dem Urteil anderer Kirchen den Wahrheiten erster Ordnung, also den Wahrheiten des Zieles, widerspricht.“ Das Problem würde sein, wie eine Kirche, die eine solche Lehre, der fundamental widersprochen wird, vertritt, sich in dieser Situation verhält: soll sie auf ein klärendes Konzil drängen oder weitere theologische Studien anregen oder diese Lehre zumindest vorläufig einklammern, oder soll sie sie, wenigstens als missverständlich, nicht länger vertreten? In jedem Fall ist eine Vergewisserung des Grundkonsens erforderlich. Angesichts der Thesen von Fries und Rahner stellen in dieser Hinsicht besonders die These IV zum Petrusdienst und die These V zur episkopalen Struktur der Kirchen eine bleibende Herausforderung dar, wenn auch hier m. E. die Materialien für einen differenzierenden Konsens bzw. wenigstens für eine In-Via-Erklärung schon erarbeitet wurden. Hilfreich erscheint mir die Einbettung in das, was die übrigen Thesen behaupten bzw. vorschlagen: die Legiti-

22 Eilert Herms/Ludomir Žak (Hg.), Grund und Gegenstand des Glaubens nach römisch-katholischer und evangelisch-lutherischer Lehre, Tübingen $2008 \mathrm{ff}$.

23 Heinrich Fries, Einigung, in: Fries/Pesch, Streiten für die eine Kirche, 54. 
mität der Teilkirchen innerhalb dieser einen Kirche (These III), der Austausch in allen ihren Lebensdimensionen“ (These VI), die Anerkennung der gegenwärtigen und künftigen Ordinationen (These VII). Für die beiden Altmeister der Ökumene mündet dies in ihre abschließende achte These: „Zwischen den einzelnen Teilkirchen besteht Kanzel- und Altargemeinschaft.“24

\section{Glauben: erfahren - bekennen - handeln}

Artikel 12 des Ökumenismusdekrets schließt dessen zweites Kapitel ab, das „die praktische Verwirklichung des Ökumenismus“ behandelt. Der Text ist also alles andere als ein frommer Abgesang, sondern so etwas wie ein ökumenisches Manifest, er wurde auch schon das „Ökumenische Hohe Lied der Liebe“ genannt. ${ }^{25}$ Während im ersten Textentwurf die praktische Ökumene im Mittelpunkt stand, verknüpft der Eingangssatz des verabschiedeten Textes, der die Basisformel des ÖRK aufgreift, Orthopraxie und Orthodoxie: „Vor allen Völkern sollen alle Christen den Glauben an den einen und dreifaltigen Gott, an den fleischgewordenen Sohn Gottes, unseren Erlöser und Herr, bekennen und in gemeinsamem Bemühen in gegenseitiger Hochschätzung Zeugnis geben für unsere Hoffnung, die nicht trügt.“ Das Bezeugen der Wahrheiten erster Ordnung, der Wahrheiten des Zieles - mit Karl Rahner spreche ich lieber von der einen Wahrheit, dem einen Geheimnis von Trinität, Inkarnation, Gnade - geschieht angesichts der Zeichen der Zeit wesentlich auch in der evangeliumsgemäßen Praxis:

Da in den heutigen Zeiten auf sozialem Gebiet die Zusammenarbeit sehr weitgehend hergestellt wird, werden ausnahmslos alle Menschen zur gemeinsamen Anstrengung gerufen, in gesteigertem Maße aber diejenigen, die an Gott glauben, am meisten aber alle Christen, da sie ja mit dem Namen Christi bezeichnet sind. Die Zusammenarbeit aller Christen drückt auf lebendige Weise jene Verbindung aus, in der sie schon untereinander geeint werden, und setzt das Antlitz Christi, des Dieners, in volleres Licht. Diese Zusammenarbeit, die bei nicht wenigen Völkern schon hergestellt ist, muss mehr und mehr vervollkommnet werden, besonders in Gebieten, wo sich die gesellschaftliche oder technische Entwicklung vollzieht, sei es bei der rechten Wertschätzung der Würde der menschlichen Person oder bei der Förderung des Guts des Friedens oder bei der Durchführung der gesellschaftlichen Anwendung des Evangeliums [so lässt sich politische Theologie bzw. Theologie der Befreiung auch umschreiben] oder bei der Weiterentwicklung von Wissenschaften und Künsten in christlichem Geist oder auch bei der Anwendung von Heilmitteln jedweder Art gegen die Nöte

24 Fries/Rahner, Einigung, 18.

25 Vgl. zum Folgenden meinen Kommentar zum Ökumenismusdekret. 
unserer Zeit, zu denen Hunger und Unglücke, Analphabetismus und Armut, Wohnungsmangel und ungerechte Verteilung der Güter gehören. ${ }^{26}$

Dies sei, so das Dekret zum Schluss, eine Möglichkeit sich besser kennenzulernen und den Weg zur Einheit zu ebnen. Das könnte als Mittel zum Zweck missverstanden werden. Deshalb möchte ich unter nochmaligem Hinweis auf die Verknüpfung am Anfang des Textes festhalten wollen: dies ist gelebtes Evangelium, dies ist ökumenisch gelebte Evangelisierung der Welt.

Erfahren, bekennen, handeln bilden keine Abfolge von Vollzügen, sondern sind miteinander verwoben. Besonders gilt es hervorzuheben, dass auch das Handeln ein Bekennen ist und dass im Handeln Erfahrungen gemacht wird, die wiederum zum Bekennen und Handeln drängen. Die Basisformel des ÖRK wurde in Neu-Delhi 1961 trinitarisch erweitert, in den Texten des letzten Konzils ist der zuvor dominierende ekklesiologische Christomonismus pneumatologisch aufgebrochen. Inzwischen kann nicht mehr von einer allgemeinen Geistvergessenheit gesprochen werden. Häufig gleicht jedoch die Erwähnung des Heiligen Geistes, der allein ja letztlich die Einheit bewirken kann, einer Vertröstung, einer taktischen Auskunft, ja einer Ausrede. Besonders den römisch-katholischen Christen ist in Erinnerung zu rufen: Es gilt darauf zu hören, was der Geist den Gemeinden sagt (so in den Sendschreiben an die sieben Gemeinden in der Apk). Es ist nicht geschrieben, darauf zu hören, was Papst und Bischöfe den Gemeinden sagen, auch nicht, was Theolog^innen den Gemeinden sagen. Das kann hilfreich sein, bleibt aber eine norma normata. Das mangelnde Vertrauen auf den Heiligen Geist kommt auch in dem Paradox zum Ausdruck, dass einerseits die Verantwortung auf ihn geschoben wird und andererseits gemeint wird, zu wissen, was der Geist von uns will. Der fromme evangelische Kabarettist Hanns Dieter Hüsch hat in seinem Pfingstpsalm formuliert, er wolle „ein Virtuose des Heiligen Geistes“ sein. Ja, das gilt es zu bezeugen in der Schwere des Seins: die Leichtigkeit des heiligenheilenden Geistes.

26 Dekret über den Ökumenismus 12,1. 
